\title{
COMPACTA WITH DENSE AMBIGUOUS LOCI OF METRIC PROJECTIONS AND ANTIPROJECTIONS
}

\author{
N. V. ZHIVKOV
}

(Communicated by J. Marshall Ash)

\begin{abstract}
In every strictly convexifiable Banach space $X$ with $\operatorname{dim} X \geq 2$ there exists a dense $G_{\delta}$ set of compacta $\mathscr{A}$ in the Hausdorff set topology such that with respect to an arbitrary equivalent strictly convex norm in $X$ both the metric projection and the metric antiprojection generated by any member of $\mathscr{A}$ are densely multivalued.
\end{abstract}

\section{INTRODUCTION}

Consider a Banach space $X$ with norm $\|\cdot\|$. Designate by $2^{X}$ the family of all non-void sets in $X$. Let $M \in 2^{X}$. The set-valued mapping $P: X \longrightarrow M$ defined by

$$
P(x, M)=\{y \in M:\|x-y\|=d(x, M)\}
$$

with distance function $d(x, M)=\inf \{\|x-z\|: z \in M\}$ is called metric projection (the nearest point mapping) generated by $M$ with respect to the norm $\|\cdot\|$, and the set-valued mapping $Q: X \longrightarrow M$ defined by

$$
Q(x, M)=\{y \in M:\|x-y\|=f(x, M)\},
$$

with farthest distance function $f(x, M)=\sup \{\|x-z\|: z \in M\}$, is a metric antiprojection (the farthest point mapping) generated by $M$ and $\|\cdot\|$.

A lot of papers have been devoted to the investigation of generic properties of metric projections and antiprojections. Far from being complete we mention works of [St], [L2], [Ko], [BF] concerning projections and [As], [L1] dealing with antiprojections. In all these works a certain "good" property of the set-valued projection (resp. antiprojection) such as existence of a best approximation (resp. existence of a farthest element), uniqueness of the solution, or well-posedness is shown to be fulfilled for the points from a residual set, i.e. a dense and $G_{\delta}$ subset of the space. It is of a natural interest then to ask what happens with the sets of "bad" points, for instance the sets whose elements fail to have unique solution for the metric projection (respectively the metric antiprojection). Following [Lu] we define ambiguous locus of a projection (resp.

Received by the editors April 19, 1994.

1991 Mathematics Subject Classification. Primary 41A65, 54E52; Secondary 46B20.

Key words and phrases. dense $G_{\delta}$, metric projection, antiprojection, ambiguous locus.

Research partially supported by the National Foundation for Scientific Research at the Bulgarian Ministry of Science and Education under contract No MM21. 
antiprojection) as the set of points at which this projection (resp. antiprojection) is multivalued, i.e. the images contain at least two different elements. In [Za] Zamfirescu showed that in a finite-dimensional Euclidean space most compacta, in the Hausdorff metric space of compacta, generate metric projections which are densely multivalued. Recently in a series of papers [BM1], [BM2], and [BM3] De Blasi and Myjak extended this result of Zamfirescu and proved various analogous theorems: In the Hausdorff space $\mathscr{B}(X)$ of bounded and closed sets most sets generate densely non-well-posed metric projections, in the spaces $\mathscr{K}(X)$ and $\mathscr{C}(X)$ of compacta and continua respectively most projections and antiprojections are densely multivalued and in the Hausdorff space $\mathscr{C} o(X)$ of convex compacta, most of them generate densely multivalued metric antiprojections. In all these theorems the Banach space $X$ is supposed to be separable and strictly convex. The aim of this work is to give a proof of the following

Theorem. In every strictly convexifiable Banach space $X$ of dimension greater than 1 there exists a dense $G_{\delta}$ set of compacta $\mathscr{A}$ in the Hausdorff set topology such that with respect to an arbitrary equivalent strictly convex norm in $X$ both the metric projection and the metric antiprojection generated by any member of $\mathscr{A}$ are densely multivalued. Moreover, the ambiguous loci of these projections and antiprojections are everywhere continual, i.e. loci's intersections with open sets in $X$ contain continuum elements.

This result is an extension of theorems of De Blasi and Myjak from [BM1] (see also [BM3]). It shows that the separability assumption can be dropped and that the set of compacta $\mathscr{A}$ is in some sense universal, i.e. it plays the same role with respect to any equivalent strictly convex norm in the space. The main construction is motivated by the construction of an example from [ $\mathrm{Zh}]$. Any compact $A \in \mathscr{A}$ is totally disconnected and has a pseudo-smooth property at each point (Lemma 3 ). This property entails multivaluedness (Lemma 2 ) since the assumption $P(\cdot, A)$ (resp. $Q(\cdot, A)$ ) is single-valued on an open set $U \subset X$ implies that $P$ (resp. $Q$ ) is a constant mapping on $U$.

\section{Preliminaries}

Suppose $(X,\|\cdot\|)$ is a strictly convex Banach space with $\operatorname{dimension} \operatorname{dim} X \geq$ 2 and $Y \subseteq X$ is a closed subspace with $\operatorname{codim} Y=2$. Suppose $\|\cdot\|_{N}$ is an equivalent norm in $Y$, different and not necessarily strictly convex, to the induced norm $\|\cdot\|$ in $X$. Consider the Banach space $X^{\prime}:=\mathbb{R}^{2} \times Y=\{x=$ $(r, s, y): r, s \in \mathbb{R}, y \in Y\}$ with norm $|\cdot|_{N}$ defined by

$$
|x|_{N}^{2}=r^{2}+s^{2}+\|y\|_{N}^{2}, \text { for } x \in X^{\prime} .
$$

Since $X$ and $X^{\prime}$ are isomorphic, it might be viewed that an equivalent norm $\|\cdot\|$ is defined in $X^{\prime}$ and from now on all the considerations will be made in $X^{\prime}$ which will be denoted as $X$ for the sake of simplicity.

The equivalence of the two norms implies existence of positive constants $\gamma_{1}$ and $\gamma_{2}$ such that

$$
\gamma_{1}|x|_{N} \leq\|x\| \leq \gamma_{2}|x|_{N} \text { whenever } x \in X
$$

The usual Euclidean norm in $\mathbb{R}^{2}$ is denoted by $|\cdot|$. We make a stipulation to identify $\mathbb{R}^{2}$ with $\mathbb{R}^{2} \times\{\theta\}$ where $\theta$ is the origin of $Y$. The following 
simple observation proves the usefulness of the norm $|\cdot|_{N}$ : The distances between elements in any two-dimensional plane which is parallel to $\mathbb{R}^{2} \times\{\theta\}$ are measured by $|\cdot|$.

In order to avoid the ambiguity in notation of functions and set-valued mappings we use the symbol of the corresponding norm, e.g., $d\left(x, M ;|\cdot|_{N}\right)$ is the distance from the element $x$ to the set $M \in 2^{X}$ with respect to the norm $|\cdot|_{N}$, while $f(\cdot, M ;\|\cdot\|)$ is the farthest distance function generated by $M$ with respect to the norm $\|\cdot\|$. Similarly, $B\left(x, \varepsilon ;|\cdot|_{N}\right)$ is the open ball with center $x$ and radius $\varepsilon$ with respect to $|\cdot|_{N}$, and $B[x, \varepsilon ;\|\cdot\|]$ stands for the closed ball with the same center and radius with respect to $\|\cdot\|$. The symbol of the norm will be dropped whenever the norm is explicitly indicated.

The Hausdorff distance for elements of $2^{X}$ with respect to $\|\cdot\|$ is defined by

$$
\begin{array}{r}
H\left(M_{1}, M_{2} ;\|\cdot\|\right):=\max \left\{\sup \left\{d\left(x, M_{1} ;\|\cdot\|\right): x \in M_{2}\right\},\right. \\
\left.\sup \left\{d\left(x, M_{2} ;\|\cdot\|\right): x \in M_{1}\right\}\right\} .
\end{array}
$$

Let $\mathscr{K}(X)$ and $\mathscr{F}(X)$ denote the families of non-empty compacta and nonempty finite subsets of $X$ respectively. Observe that $\mathscr{K}(X)$ equipped with a Hausdorff distance is a complete metric space and $\mathscr{F}(X)$ is dense in it. Besides, the different Hausdorff distances generated by equivalent norms in $X$ do generate one and the same set topology which is designated by $\mathscr{H}$. For balls in a Hausdorff metric space the symbol $\mathscr{O}$ is used. Let $K \in \mathscr{K}(X)$. It follows immediately from (1):

$$
\mathscr{O}\left(K, \gamma_{1} \varepsilon ;\|\cdot\|\right) \subset \mathscr{O}\left(K, \varepsilon ;|\cdot|_{N}\right) \subset \mathscr{O}\left(K, \gamma_{2} \varepsilon ;\|\cdot\|\right) .
$$

A subset $M \in 2^{X}$ is called totally disconnected whenever $M$ contains at least two different elements and for every $x$ and $y$ from $X, x \neq y$, there is no continuous curve in $M$ with endpoints $x$ and $y$, i.e. there is no continuous map $\phi:[0,1] \longrightarrow M$ such that $\phi(0)=x$ and $\phi(1)=y$.

A subset $M \in 2^{X}$ is called $\varepsilon$-disconnected (with respect to $|\cdot|_{N}$ ) whenever $M$ contains at least two distant elements at greater than $\varepsilon$ distance and for every $x$ and $y$ in $M$ with $|x-y|_{N}>\varepsilon$ there is no continuous curve in $M$ with endpoints $x$ and $y$.

Obviously, $M \in 2^{X}$ is totally disconnected, if and only if $M$ is $\varepsilon$-disconnected for every positive $\varepsilon$. Also, the notion of total disconnectedness is purely topological.

For the elements of $\mathscr{F}(X)$ define a separator function (with respect to $|\cdot|_{N}$ ):

$$
\operatorname{sep} F=\min \left\{1,\left\{|x-y|_{N}: x, y \in F, x \neq y\right\}, \quad F \in \mathscr{F}(X) .\right.
$$

The value of $\operatorname{sep} F$ is 1 whenever $F$ is a singleton.

Lemma 1. Let $K_{1}$ and $K_{2}$ be two disjoint and non-void compacta in the Banach space $(X,\|\cdot\|)$ and $x \in X$ be an element such that $d\left(x, K_{1}\right)=d\left(x, K_{2}\right)$ (respectively $\left.f\left(x, K_{1}\right)=f\left(x, K_{2}\right)\right)$. Then an arbitrary neighborhood of $x$ contains continually many elements with the same property.

Proof. Projection part. Suppose $y_{i} \in P\left(x, K_{i}\right), i=1,2$. Denote $y_{i}(t)=(1-$ $t) x+t y_{i}$ for $t \in(0,1]$. Obviously, $y_{i} \in P\left(y_{i}(t), K_{i}\right), i=1,2$. Make use of the continuity principle applied to the function $\phi_{t}(u)=d\left(u, K_{1}\right)-d\left(u, K_{2}\right)$ defined on $\left[y_{1}(t), y_{2}(t)\right]$ since $\phi\left(y_{1}(t)\right) \leq 0, \phi\left(y_{2}(t)\right) \geq 0$. There is $y(t) \in\left[y_{1}(t), y_{2}(t)\right]$ 


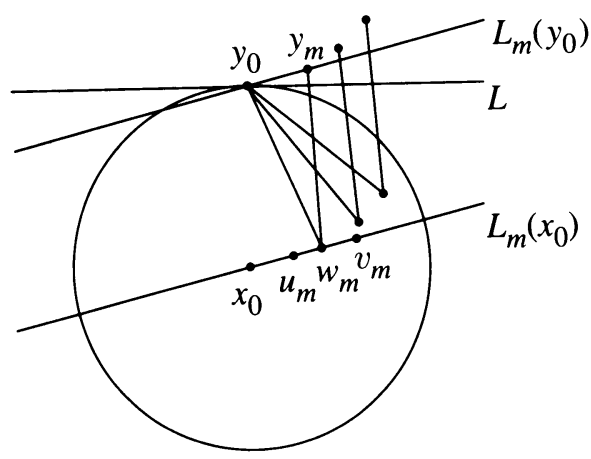

FIGURE 1

such that $d\left(y(t), K_{1}\right)=d\left(y(t), K_{2}\right)$. For different $t$ the values of $y(t)$ are different and form a set with the power of continuum.

Antiprojection part. Suppose $y_{i} \in Q\left(x, K_{i}\right), i=1,2$, and define $y_{i}(t)=$ $(1-t) x+t y_{i}$ for $t<0$. Then $y_{i} \in Q\left(y_{i}(t), K_{i}\right), i=1,2$, and the continuity principle is applied to the function $\psi_{t}(u)=f\left(u, K_{1}\right)-f\left(u, K_{2}\right)$ where $u \in$ $\left[y_{1}(t), y_{2}(t)\right]$ since $\psi\left(y_{1}(t)\right) \geq 0, \psi\left(y_{2}(t)\right) \leq 0$.

Lemma 2. Let $(X,\|\cdot\|)$ be a strictly convex Banach space and $x_{0}, y_{0} \in X$ be such that $\left\|x_{0}-y_{0}\right\|=d>0$. Suppose $L=\left\{y=y_{0}+\right.$ te $\left.: t \in \mathbb{R}\right\}$ where $\|e\|=1$ is a line such that $\left\{y_{0}\right\}=L \cap B\left[x_{0}, d\right]$, and there is a sequence $\left(y_{m}\right)$ satisfying

(i) $\lim y_{m}=y_{0}$,

(ii) $\lim d\left(y_{m}, L\right) /\left\|y_{m}-y_{0}\right\|=0$.

Then there exists a sequence $\left(w_{m}\right), \quad \lim w_{m}=x_{0}$ such that $\left\|y_{0}-w_{m}\right\|=$ $\left\|y_{m}-w_{m}\right\|$.

Proof. Denote $e_{m}=\left(y_{m}-y_{0}\right) /\left\|y_{m}-y_{0}\right\|$, and define the lines $L_{m}\left(y_{0}\right)=\{y=$ $\left.y_{0}+\lambda e_{m}: \lambda \in \mathbb{R}\right\}$ and $L_{m}\left(x_{0}\right)=\left\{x=x_{0}+\lambda e_{m}: \lambda \in \mathbb{R}\right\}$ and the sequences $\left(u_{m}\right)$ and $\left(v_{m}\right)$ such that $\left\{u_{m}\right\}=P\left(y_{0}, L_{m}\left(x_{0}\right) ;\|\cdot\|\right), \quad\left\{v_{m}\right\}=P\left(y_{m}, L_{m}\left(x_{0}\right) ;\|\cdot\|\right)$. Moreover, $\left\{y_{0}\right\}=P\left(u_{m}, L_{m}\left(y_{0}\right) ;\|\cdot\|\right), \quad\left\{y_{m}\right\}=P\left(v_{m}, L_{m}\left(y_{0}\right) ;\|\cdot\|\right)$ and

$$
\left\|y_{0}-y_{m}\right\|=\left\|u_{m}-v_{m}\right\| \text {. }
$$

It follows that $\left\|y_{0}-u_{m}\right\|<\left\|y_{m}-u_{m}\right\|$ and $\left\|y_{m}-v_{m}\right\|<\left\|y_{0}-v_{m}\right\|$. Apply the continuity principle to the function $\phi_{m}(w)=\left\|y_{0}-w\right\|-\left\|y_{m}-w\right\|, w \in$ $\left[u_{m}, v_{m}\right]$, in order to show the existence of $w_{m} \in\left(u_{m}, v_{m}\right)$ such that

$$
\left\|y_{0}-w_{m}\right\|=\left\|y_{m}-w_{m}\right\|
$$

as indicated in Figure 1.

It is to be proved that $\lim u_{m}=x_{0}$. Indeed, $u_{m}=x_{0}+\lambda_{m} e_{m}, \lambda_{m} \in \mathbb{R}$. Since $\left\|u_{m}-y_{0}\right\|<\left\|x_{0}-y_{0}\right\|$, then $\left\|u_{m}-x_{0}\right\|=\left|\lambda_{m}\right| \leq 2 d$, i.e. the sequence of reals $\left(\lambda_{m}\right)$ is bounded. Let $\lambda_{0}$ be a cluster point. On the other hand (ii) implies that $\left(e_{m}\right)$ has at least one and at most two cluster points $e$ and $-e$. Let $e$ be so, the other case is treated similarly. Then $u_{0}=x_{0}+\lambda_{0} e$ is a cluster point of $\left(u_{m}\right)$. If we assume that $u_{0} \neq x_{0}$, then for the strict convexity of $\|\cdot\|$ and $P\left(x_{0}, L ;\|\cdot\|\right)=\left\{y_{0}\right\}$ it follows that $\left\|x_{0}-y_{0}\right\|<\left\|u_{0}-y_{0}\right\|$. There exists then $\delta>0, \quad \delta<\left\|u_{0}-x_{0}\right\|$, such that $\left\|x_{0}-y_{0}\right\|<\left\|u-y_{0}\right\|$ whenever $u \in B\left(u_{0}, \delta ;\|\cdot\|\right)$. The last inequality contradicts the choice of $\left(u_{m}\right)$, since for infinitely many values of $m\left\|x_{0}-y_{0}\right\|<d\left(y_{0}, L_{m}\left(x_{0}\right) ;\|\cdot\|\right)$ and $u_{m} \neq x_{0}$. 
Hence $\lambda_{0}=0$ and $x_{0}$ is the only cluster point of $\left(u_{m}\right)$. According to (2) and (i) it follows that $\lim v_{m}=\lim w_{m}=x_{0}$.

\section{Proof of The MAIN Result}

It suffices to prove the statement of the theorem with the norm $\|\cdot\|$. The proof is partitioned in six steps.

The first step is a definition of a set $\mathscr{A}$ : Designate by $V_{n}$ the set of vertices of a regular $2^{n}$-gon inscribed in the unit circumference in $\mathbb{R}^{2}$, i.e.

$$
V_{n}=\left\{\left(\cos \left(i \pi / 2^{n-1}\right), \sin \left(i \pi / 2^{n-1}\right), \theta\right): i=0,1, \ldots, 2^{n}-1 ; \theta \in Y\right\}, n \geq 3 \text {. }
$$

It is a routine matter to verify that

$$
\operatorname{sep} V_{n}=2 \sin \left(\pi / 2^{n}\right) \text {. }
$$

Assign $\quad \mathscr{U}_{n}:=\bigcup\left\{\mathscr{O}\left(F+n^{-1} \operatorname{sep}(F) V_{n}, n^{-1} \operatorname{sep}(F) \sigma_{n} ;|\cdot|_{N}\right): F \in \mathscr{F}(X)\right\}$, with $\sigma_{n}=\sin ^{2}\left(\pi / 2^{n}\right), \quad n \geq 3$, and define

$$
\mathscr{A}:=\bigcap_{n=3}^{\infty} \mathscr{U}_{n} .
$$

Obviously $\mathscr{U}_{n}$ are open sets as unions of open balls. On the other hand all elements from the type $F+n^{-1} \operatorname{sep} F$ for $F \in \mathscr{F}(X)$ and $n \geq 3$ form a dense subset of $\mathscr{F}(X)$ in the $\mathscr{H}$-topology, and since $\mathscr{F}(X)$ is dense in $\mathscr{K}(X)$, then $\mathscr{U}_{n}$ are open and dense. Hence $\mathscr{A}$ is a dense $G_{\delta}$ subset of $(\mathscr{K}(X), \mathscr{H})$.

The second step of the proof is to show that any element $A$ of $\mathscr{A}$ is a totally disconnected set. For that reason let $A \in \mathscr{A}$ and fix $n \geq 3$. There is a finite set $F$ with $k$ different elements, $k \geq 1$, such that

$$
H\left(A, F+n^{-1} \operatorname{sep}(F) V_{n} ;|\cdot|_{N}\right)<n^{-1} \operatorname{sep}(F) \sigma_{n} .
$$

Having in mind (4) we get

$$
\operatorname{sep}\left(F+n^{-1} \operatorname{sep}(F) V_{n}\right)=2 n^{-1} \operatorname{sep}(F) \sqrt{\sigma_{n}} .
$$

It is seen from (5) and (6) that $A$ might be viewed as union of $k 2^{n}$ disjoint compacta $A_{i}$,

$$
A=\bigcup_{i=1}^{k 2^{n}} A_{i}
$$

such that for $y_{1} \in A_{i}$ and $y_{2} \in A_{j}$

$$
\left|y_{1}-y_{2}\right|_{N}>2 n^{-1} \operatorname{sep}(F)\left(\sqrt{\sigma_{n}}-\sigma_{n}\right), \quad \text { iff } i \neq j,
$$

which implies that $A$ is an $\varepsilon_{n}$-disconnected set, for $\varepsilon_{n}=2 n^{-1}\left(\sqrt{\sigma_{n}}-\sigma_{n}\right)$. Now let $n$ go to infinity.

For the third step a "tangent" property of the elements of $\mathscr{A}$ is needed.

Lemma 3. Suppose $A \in \mathscr{A}$ and $y_{0} \in A$. There exists a line $L=\left\{y=y_{0}+\lambda e\right.$ : $\lambda \in \mathbb{R}\},\|e\|=1$, and a sequence $\left(y_{m}\right)$ in $A$ such that

(i) $\lim y_{m}=y_{0}$,

(ii) $\lim d\left(y_{m}, L ;\|\cdot\|\right) /\left\|y_{m}-y_{0}\right\|=0$,

(iii) the sequence $\left(\left(y_{m}-y_{0}\right) /\left\|y_{m}-y_{0}\right\|\right)$ has two cluster points $e$ and $-e$. 


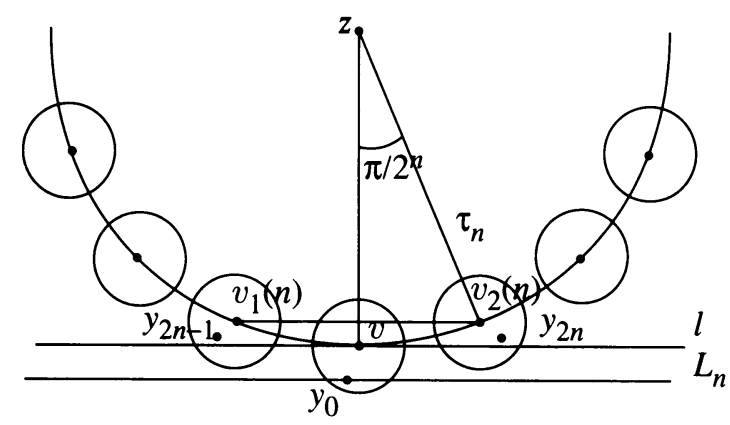

Figure 2

Proof. For every $n \geq 3$ there is $F \in \mathscr{F}(X)$ satisfying (5). Set for convenience $\tau_{n}=n^{-1} \operatorname{sep} F$. There exist $z$ and $v, z \in F$ and $v \in z+\tau_{n} V_{n}$, such that $\left|y_{0}-v\right|_{N}<\tau_{n} \sigma_{n}$. Denote by $v_{1}(n)$ and $v_{2}(n)$ the two neighboring (and nearest with respect to $\left.|\cdot|_{N}\right)$ to $v$ elements from $z+\tau_{n} V_{n}$. It follows from (6) that

$$
\left|v-v_{i}(n)\right|_{N}=2 \tau_{n} \sqrt{\sigma_{n}}, \quad i=1,2 .
$$

Designate by $l$ the line in the plane $z+\mathbb{R}^{2}$, passing through $v$ and parallel to the segment $\left[v_{1}(n), v_{2}(n)\right]$. After elementary calculations we get

$$
\begin{aligned}
d\left(v,\left[v_{1}(n), v_{2}(n)\right] ;|\cdot|_{N}\right) & =\tau_{n}\left(1-\cos \left(\pi / 2^{n-1}\right)\right) \\
& =d\left(v_{i}(n), l ;|\cdot|_{N}\right), \quad i=1,2 .
\end{aligned}
$$

Choose in accordance to (5) $y_{2 n-1} \in A \cap B\left(v_{1}(n), \tau_{n} \sigma_{n} ;|\cdot|_{N}\right)$ and $y_{2 n} \in$ $A \cap B\left(v_{2}(n), \tau_{n} \sigma_{n} ;|\cdot|_{N}\right)$. Assign $e_{n}:=\left(v_{2}(n)-v_{1}(n)\right) /\left\|v_{2}(n)-v_{1}(n)\right\|$ and $L_{n}:=\left\{y=y_{0}+\lambda e_{n}: \lambda \in \mathbb{R}\right\}$; see Figure 2 .

Since all the members of the sequence $\left(e_{n}\right)$ belong to $\mathbb{R}^{2}$, then it has a cluster point $e, \quad\|e\|=1$, which is a limit of a subsequence $\left(e_{n_{k}}\right)$. Denote $L=\left\{y=y_{0}+\lambda e: \lambda \in \mathbb{R}\right\}$ and $\eta_{n}=\left\|e_{n}-e\right\|$.

The triangle inequality and (9) imply

$$
2 \tau_{n}\left(\sqrt{\sigma_{n}}-\sigma_{n}\right) \leq\left|y_{2 n-j}-y_{0}\right|_{N} \leq 2 \tau_{n}\left(\sqrt{\sigma_{n}}+\sigma_{n}\right), \quad j=0,1 .
$$

Moreover

$$
\begin{aligned}
\left|y_{2 n}-y_{2 n-1}\right|_{N} & \geq\left|v_{2}(n)-v_{1}(n)\right|_{N}-2 \tau_{n} \sigma_{n} \\
& =2 \tau_{n} \sqrt{\sigma_{n}}\left(2 \cos \left(\pi / 2^{n}\right)-\sqrt{\sigma_{n}}\right) .
\end{aligned}
$$

It follows from (1), (5), (10) and (11) that (13)

$$
\begin{aligned}
\frac{d\left(y_{2 n-j}, L_{n} ;\|\cdot\|\right)}{\left\|y_{2 n-j}-y_{0}\right\|} & \leq \frac{\gamma_{2}}{\gamma_{1}} \cdot \frac{d\left(y_{2 n-j}, L_{n} ;|\cdot|_{N}\right)}{\left|y_{2 n-j}-y_{0}\right|_{N}} \\
& \leq \frac{\gamma_{2}}{\gamma_{1}} \cdot \frac{1-\cos \left(\pi / 2^{n-1}\right)+2 \sigma_{n}}{2\left(\sqrt{\sigma_{n}}-\sigma_{n}\right)}=\frac{\gamma_{2}}{\gamma_{1}} \cdot \frac{2 \sqrt{\sigma_{n}}}{1-\sqrt{\sigma_{n}}}, \quad j=0,1 .
\end{aligned}
$$

Let now $y \in X, \quad y \neq y_{0}$. In order to evaluate the distance from $y$ to the line $L$ by making use of the distances from $y$ to $L_{n}$, pick $w_{n} \in P\left(y, L_{n} ;\|\cdot\|\right)$ and consider the inequalities:

$$
\begin{aligned}
d(y, L ;\|\cdot\|) & \leq\left\|y-w_{n}\right\|+d\left(w_{n}, L ;\|\cdot\|\right) \\
& \leq\left\|y-w_{n}\right\|+\left\|w_{n}-y_{0}\right\| .\left\|e_{n}-e\right\| \\
& \leq\left(1+\eta_{n}\right) d\left(y, L_{n} ;\|\cdot\|\right)+\eta_{n}\left\|y-y_{0}\right\| .
\end{aligned}
$$


Redefine a sequence $\left(y_{m}\right)$ with abuse of notation:

$$
y_{m}= \begin{cases}y_{2 n_{k}-1} & \text { for } m=2 k-1 \\ y_{2 n_{k}} & \text { for } m=2 k\end{cases}
$$

herein $k$ is the integer part of $(m+1) / 2$.

The statement (i) follows from (1) and (11):

$$
\left\|y_{m}-y_{0}\right\| \leq \gamma_{2}\left|y_{m}-y_{0}\right|_{N} \leq 2 \gamma_{2} \tau_{n_{k}}\left(\sqrt{\sigma_{n_{k}}}+\sigma_{n_{k}}\right)<2 \gamma_{2} n_{k}^{-1} .
$$

In order to get (ii) compare (13) with (14)

$$
\begin{aligned}
d\left(y_{m}, L ;\|\cdot\|\right) /\left\|y_{m}-y_{0}\right\| & \leq\left(1+\eta_{n_{k}}\right) d\left(y_{m}, L ;\|\cdot\|\right) /\left\|y_{m}-y_{0}\right\|+\eta_{n_{k}} \\
& \leq 2 \gamma_{2} \gamma_{1}^{-1}\left(1+\eta_{n_{k}}\right) \sqrt{\sigma_{n_{k}}} /\left(1-\sqrt{\sigma_{n_{k}}}\right)+\eta_{n_{k}} .
\end{aligned}
$$

The expression from the right side of the last inequality tends to 0 when $m$ increases unboundedly.

It remains to prove (iii). Set $u_{m}=y_{0}+\left(y_{m}-y_{0}\right) /\left\|y_{m}-y_{0}\right\|$. The statement (ii) is equivalent with $\lim d\left(u_{m}, L ;\|\cdot\|\right)=0$. The local compactness of $L$ entails existence of a cluster point $u=y_{0}+\lambda e$ for $\left(u_{m}\right)$. It follows from the continuity of $\|\cdot\|$ that $|\lambda|=1$, i.e. either $u=y_{0}+e$ or $u=y_{0}-e$. Now, it is to be shown that $\left(u_{m}\right)$ is not convergent, whence both $y_{0}+e$ and $y_{0}-e$ are cluster points. To reach this goal it suffices to prove that $\left(u_{m}^{\prime}\right)$, with $u_{m}^{\prime}=y_{0}+\left(y_{m}-y_{0}\right) / \nu_{m}$ and $\nu_{m}=\left|y_{m}-y_{0}\right|_{N}$, is not a convergent sequence:

$$
\left|u_{2 k}^{\prime}-u_{2 k-1}^{\prime}\right|_{N}=\left|\nu_{2 k}^{-1}\left(y_{2 k}-y_{2 k-1}\right)+\left(\nu_{2 k}^{-1}-\nu_{2 k-1}^{-1}\right)\left(y_{2 k-1}-y_{0}\right)\right|_{N}
$$

and after applying (11) and (12)

$$
\begin{aligned}
& \geq \nu_{2 k}^{-1}\left(\left|y_{2 k}-y_{2 k-1}\right|_{N}-\left|\nu_{2 k}-\nu_{2 k-1}\right|\right) \geq \nu_{2 k}^{-1}\left(\left|y_{2 k}-y_{2 k-1}\right|_{N}-4 \tau_{n_{k}} \sigma_{n_{k}}\right) \\
& \geq\left(2 \cos \left(\pi / 2^{n_{k}}\right)-3 \sqrt{\sigma_{n_{k}}}\right) /\left(1+\sqrt{\sigma_{n_{k}}}\right) .
\end{aligned}
$$

The last expression tends to 2 with $k$ going to infinity. The proof of Lemma 3 is completed.

Continue the proof of the theorem. The fourth step is to show that for given $A \in \mathscr{A}$ there is a dense subset of $X$ whose elements have at least two best approximations for the projection mapping $P(\cdot, A ;\|\cdot\|)$. Assume the contrary. There exist $x_{0} \in X$ and $\varepsilon>0$ such that $P(\cdot, A ;\|\cdot\|)$ is singlevalued in the ball $B\left(x_{0}, \varepsilon ;\|\cdot\|\right)$. Therefore all elements of this ball are projected onto a single point $y_{0} \in A$. Indeed, if $x_{1}, x_{2} \in B\left(x_{0}, \varepsilon ;\|\cdot\|\right), x_{1} \neq x_{2}$, and $\left\{y_{i}\right\}=P\left(x_{i}, A ;\|\cdot\|\right), i=1,2$, then the upper semicontinuity of $P(\cdot, A ;\|\cdot\|)$ [Si] and its single-valuedness imply the existence of a continuous curve $\kappa:=$ $\left\{y \in A:\{y\}=P\left(t x_{2}+(1-t) x_{1}, A ;\|\cdot\|\right), 0 \leq t \leq 1\right\}, \kappa(0)=y_{1}, \kappa(1)=y_{2}$ connecting $y_{1}$ and $y_{2}$, whence $y_{1}=y_{2}$ as $A$ is totally disconnected.

Assume now, with no loss of generality, that $\left\|x_{0}-y_{0}\right\|=d>0$ since int $A=$ $\varnothing$, and let $L$ be the line through $y_{0}$ satisfying (i), (ii) and (iii) from Lemma 3 for a sequence $\left(y_{m}\right)$. In order to apply Lemma 2 we need only prove that $L$ is a supporting line for $B\left[x_{0}, d ;\|\cdot\|\right]$. Suppose this is not true, i.e. there exists $x \in L \cap B\left(x_{0}, d ;\|\cdot\|\right)$. Then for some $\delta>0, \operatorname{co}\left(B(x, \delta ;\|\cdot\|) \cup\left\{y_{0}\right\}\right) \subset$ $B\left(x_{0}, d ;\|\cdot\|\right) \cup\left\{y_{0}\right\}$ (here co stands for the convex hull of a set). It follows from (iii) that $B\left(x_{0}, d ;\|\cdot\|\right)$ contains elements from $A$ and $y_{0}$ is not a best approximation, which is a contradiction. Hence $\left\{y_{0}\right\}=L \cap B\left[x_{0}, d ;\|\cdot\|\right]$. 
To conclude the fourth step of the proof apply Lemma 2. There is a sequence $\left(w_{m}\right)$ such that $\lim w_{m}=x_{0}$ and (3) holds: $\left\|y_{0}-w_{m}\right\|=\left\|y_{m}-w_{m}\right\|$. Therefore, for large $m$ the point $w_{m}$ is in the $\varepsilon$-neighborhood of $x_{0}$ and $P(\cdot, A ;\|\cdot\|)$ is multivalued at $w_{m}$ since $y_{0}, y_{m} \in A$ and $y_{0} \neq y_{m}$. Thus, the assumption for single-valuedness of $P$ in $B\left(x_{0}, \varepsilon ;\|\cdot\|\right)$ leads to a contradiction.

The next fifth step is to show that the ambiguous locus of a projection generated by arbitrary $A \in \mathscr{A}$ is everywhere uncountable (in fact everywhere continual). Let $x_{0} \in X$ and $y_{1}, y_{2} \in P\left(x_{0}, A ;\|\cdot\|\right),\left\|y_{1}-y_{2}\right\|=r>0$. Choose $n$ sufficiently large so that $n^{-1}\left(\sqrt{\sigma_{n}}-\sigma_{n}\right)<r / 2 \gamma_{2}$. There is $F \in \mathscr{F}(X)$ such that (5), (7) and (8) hold. Since $2 n^{-1} \operatorname{sep}(F)\left(\sqrt{\sigma_{n}}-\sigma_{n}\right)<r \gamma_{2}^{-1} \leq\left|y_{1}-y_{2}\right|_{N}$, then by (8) there are $i$ and $j, i \neq j$, such that $y_{1} \in A_{i}$ and $y_{2} \in A_{j}$. The set $A$ might be represented as the union of two disjoint compacta $K_{1}$ and $K_{2}$. It remains to apply Lemma 1.

The final part of the proof concerns metric antiprojections. We proceed in an analogous way. The assumption that there is $A \in \mathscr{A}$ such that $Q(\cdot, A ;\|\cdot\|)$ is single-valued in some open set leads to the conclusion that $Q$ maps all the elements from this open set onto a singleton, because an antiprojection generated by a compact is upper semicontinuous [ $\mathrm{Bl}$ ], and $A$ is totally disconnected. Further, apply consecutively Lemmata 3,2 , and 1 (the antiprojection part). The proof of the theorem is completed.

Remarks. The theorem implies that the convex compacta which generate densely multivalued metric antiprojections are dense in $\mathscr{C} o(X)$, the Hausdorff space of convex compacta, but whether they form a $G_{\delta}$ set too is an open question. We note also, that it is not known whether the separability assumption can be relaxed for Hausdorff spaces different from $\mathscr{K}(X)$.

Added note. After this paper was submitted some new results appeared: In [BKM] De Blasi, Kenderov, and Myjak proved that most sets in the Hausdorff space $\mathscr{S}(X)$ of star-shaped compacta in a separable strictly convex Banach space $X$ generate densely multivalued metric projections, and in [Zh2] Peano continua of integer Hausdorff dimension which satisfy the statement of the present theorem for metric projections are constructed.

\section{REFERENCES}

[As] E. Asplund, Farthest points in reflexive locally uniformly rotund spaces, Israel J. Math. 4 (1966), 213-216.

[Bl] J. Blatter, Weiteste Punkte und Nachste Punkte, Rev. Roumaine Math. Pures Appl. 14 (1969), 615-621.

[BKM] F.S. De Blasi, P.S. Kenderov, and J. Myjak, Ambiguous loci of the metric projection onto compact starshaped sets in a Banach space, Monatsh. Math. 119 (1995), 23-36.

[BM1] F.S. De Blasi and J. Myjak, Ambiguous loci of the nearest point mapping in Banach spaces, Arch. Math. 61 (1993), 377-384.

[BM2] _ Ambiguous loci of the farthest distance mapping from compact convex sets, Studia Math. (to appear).

[BM3] __ On compact connected sets in Banach spaces (to appear).

[BF] J.M. Borwein and S. Fitzpatrick, Existence of nearest points in Banach spaces, Canad. J. Math. 41 (1989), 702-720.

[Ko] S.V. Konyagin, On approximation of closed sets in Banach spaces and the characterization of strongly convex spaces, Soviet Math. Dokl. 21 (1980), 418-422. 
[L1] Ka-Sing Lau, Farthest points in weakly compact spaces, Israel J. Math. 22 (1975), 168-174.

[L2] _ Almost Chebyshev subsets in reflexive Banach spaces, Indiana Univ. Math. J. 27 (1978), 791-795.

[Lu] D. Lubell, Proximity, Swiss cheese and offshore rights, preprint.

[Si] I. Singer, Some remarks on approximative compactness, Rev. Roumaine Math. Pures Appl. 9 (1964), 167-177.

[St] S.B. Stechkin, Approximative properties of subsets of Banach spaces, Rev. Roumaine Math. Pures Appl. 8 (1963), 5-8.

[Za] T. Zamfirescu, The nearest point mapping is single-valued nearly everywhere, Arch. Math. 54 (1990), 563-566.

[Zh] N.V. Zhivkov, Examples of plane compacta with dense ambiguous loci, C. R. Acad. Bulgare Sci. 46 (1993), 27-30.

[Zh2] , Peano continua generating densely multivalued metric projections, Rend. Sem. Mat. Univ. Politec. Torino (to appear).

Institute of Mathematics, Bulgarian ACademy of Sciences, Sofia 1113, Bulgaria

E-mail address: nickor@bgearn.bitnet 